\title{
EMSS: New EM-type algorithms for the Heckman selection model in $\mathbf{R}$
}

by Kexuan Yang, Sang Kyu Lee, Jun Zhao and Hyoung-Moon Kim

\begin{abstract}
When investigators observe non-random samples from populations, sample selectivity problems may occur. The Heckman selection model is widely used to deal with selectivity problems. Based on the EM algorithm, Zhao et al. (2020) developed three algorithms, namely, ECM, ECM(NR), and ECME(NR), which also have the EM algorithm's main advantages: stability and ease of implementation. This paper provides the implementation of these three new EM-type algorithms in the package EMSS and illustrates the usage of the package on several simulated and real data examples. The comparison between the maximum likelihood estimation method (MLE) and three new EM-type algorithms in robustness issues is further discussed.
\end{abstract}

\section{Introduction}

The problem arising from the sampling mechanism where an investigator extracts a sample nonrandomly, and then this sample cannot represent the population is usually referred to as a sample selection problem. Methods relying on a distributional assumption are widely used to deal with this selection problem. A classical sample selection model under the assumption of bivariate normality is introduced in Heckman (1974), and it is commonly called the Heckman selection model. Heckman (1979) further developed two estimation procedures for the above Heckman selection model: the maximum likelihood estimation method (MLE) and the two-step method.

The application of these two methods in the Heckman selection model is first described in the R package sampleSelection by Toomet and Henningsen (2008). For the observations where outlying ones are considered in the Heckman selection model, Zhelonkin et al. (2016) found that the unboundedness of the influence functions in the two-step method leads to an arbitrary bias. Zhelonkin et al. (2016) developed a robust two-stage method that performs more robustly than the two-step method, and the ssmrob package is available for robust estimation and inference for the selection model.

Little and Rubin (2002, pp. 322-323) applied an EM algorithm, which is numerically stable and easily implemented, to estimate the parameters of the Heckman selection model. However, it is limited to the cases in which the two vectors of the observed characteristics in the Heckman selection model are the same. Zhao et al. (2020) extended three new EM-type algorithms: expectation-conditional maximization (ECM), expectation-conditional maximization with Newton-Raphson method (ECM(NR)), and Expectation/Conditional Maximization Either (ECME) to more general cases. They also have the main advantages of the EM algorithm, namely stability and ease of implementation. However, section 6 in Zhao et al. (2020) suggests that the ECME algorithms require much more time than other two EM-type algorithms in the same real data analysis. In addition, there is still no R package available for these EM-type algorithms. Meng and Rubin (1993), Liu and Rubin (1994), and McLachlan and Krishnan (2008) are helpful to understand the procedures of the ECM and ECME algorithms.

This study developed the ECME algorithm by first applying the Newton-Raphson method to reduce the estimation time. Then, it is proposed to describe these new EM-type algorithms in R. In the next section, the Heckman selection model is described in brief, followed by new algorithms, namely ECM, ECM(NR), and ECME. Next, the usage of the EMSS package is presented through simulation and real data examples. Then, the robustness issue is further discussed for the MLE method and the new EM-type algorithms. Under the conditions where the robustness issue arises from the initial values, the EMSS package is preferable to the sampleSelection. Because of the unreasonable results in the MLE or the two-step method, the "NA" might occur in the standard errors in the sampleSelection. However, the standard errors can be calculated effectively in the EMSS package in almost all cases. Finally, we provide a summary of this study.

The EMSS package is available from the Comprehensive R Archive Network (CRAN) at https: //CRAN. R-project.org/package=EMSS and the GitHub at https://github.com/SangkyuStat/EMSS. R code for the examples demonstrated herein has been provided as supplementary material. The supplementary code has been tested with EMSS version 1.1.1, and results presented herein have been produced with this version. 


\section{Model and algorithms}

\section{Heckman selection model}

Suppose that the regression model of the outcome variable of interest is

$$
Y_{i 1}=\mathbf{x}_{i}^{\top} \boldsymbol{\beta}+\epsilon_{i}, \quad i=1, \ldots, N .
$$

Due to selection mechanism,

$$
Y_{i 2}=\mathbf{w}_{i}^{\top} \gamma+\eta_{i}, \quad i=1, \ldots, N,
$$

we observe only $N_{1}$ out of $N$ observations $y_{i 1}$ for which $y_{i 2}>0$ such that

$$
u_{i}=I\left(y_{i 2}>0\right) \text {. }
$$

$\mathbf{x}_{i} \in \mathbb{R}^{p}$ and $\mathbf{w}_{i} \in \mathbb{R}^{q}$ are observed characteristics. In addition, vectors $\beta \in \mathbb{R}^{p}, \gamma \in \mathbb{R}^{q}$ are unknown parameters. Assume that the error terms $\epsilon_{i}$ and $\eta_{i}$ follow bivariate normality as in Heckman (1974), that is,

$$
\left(\begin{array}{c}
\epsilon_{i} \\
\eta_{i}
\end{array}\right) \stackrel{\text { i.i.d. }}{\sim} N_{2}\left[\left(\begin{array}{l}
0 \\
0
\end{array}\right),\left(\begin{array}{cc}
\sigma^{2} & \rho \sigma \\
\rho \sigma & 1
\end{array}\right)\right],
$$

where "i.i.d." means independent and identically distributed, then $\left(Y_{i 1}, Y_{i 2}\right)$ also follow bivariate normal distribution.

\section{EM-type algorithms}

\section{ECM and ECM(NR) algorithms}

The ECM algorithm is discussed first. In the Heckman selection model, it is assumed that we observe the first $N_{1}$ out of $N y_{i 1}$ observations. The observed data are $\mathbf{y}_{o b s}=\left(y_{11}, \cdots, y_{N_{1}, 1}\right)^{\top}$, and the missing data are $\mathbf{y}_{\text {mis }}=\left(\left(y_{N_{1}+1,1}, \cdots, y_{N, 1}\right), \mathbf{y}_{2}^{\top}\right)^{\top}$. Applying the invariance property of MLEs, the parameter $\boldsymbol{\theta}=\{\boldsymbol{\beta}, \gamma, \sigma, \rho\}$ can be transformed to $\boldsymbol{\theta}^{*}=\left\{\boldsymbol{\beta}, \boldsymbol{\gamma}, \psi^{*}, \rho^{*}\right\}$ with $\psi=\sigma^{2}\left(1-\rho^{2}\right), \psi^{*}=\log (\psi), \rho^{*}=\rho \sigma$ and

$$
\sigma^{2}=\exp \left(\psi^{*}\right)+\left(\rho^{*}\right)^{2} \text { and } \rho=\frac{\rho^{*}}{\sqrt{\exp \left(\psi^{*}\right)+\left(\rho^{*}\right)^{2}}} .
$$

The complete data log-likelihood is

$$
\begin{aligned}
& l_{c}\left(\boldsymbol{\theta}^{*} \mid \mathbf{y}\right)=-N \log (2 \pi)-\frac{N}{2} \log (\psi)-\frac{1}{2 \psi}\left\{\sum_{i=1}^{N}\left(y_{i 1}-\mathbf{x}_{i}^{\top} \boldsymbol{\beta}\right)^{2}\right\} \\
& -\frac{1}{2}\left(1+\frac{\left(\rho^{*}\right)^{2}}{\psi}\right)\left\{\sum_{i=1}^{N}\left(y_{i 2}-\mathbf{w}_{i}^{\top} \gamma\right)^{2}\right\}+\frac{\rho^{*}}{\psi}\left\{\sum_{i=1}^{N}\left(y_{i 1}-\mathbf{x}_{i}^{\top} \boldsymbol{\beta}\right)\left(y_{i 2}-\mathbf{w}_{i}^{\top} \gamma\right)\right\} .
\end{aligned}
$$

The corresponding Q-function, which is the conditional expectation of the above complete-data loglikelihood $l_{c}\left(\boldsymbol{\theta}^{*} \mid \mathbf{y}\right)$ with respect to the conditional distribution of $\mathbf{y}_{\text {mis }}$ given $\mathbf{y}_{o b s}$ at the $k$-th iteration, is obtained as

$$
\begin{gathered}
Q\left(\boldsymbol{\theta}^{*} \mid \hat{\boldsymbol{\theta}}^{*(k)}\right)=E\left[l_{c}\left(\boldsymbol{\theta}^{*} \mid \mathbf{y}\right) \mid \hat{\boldsymbol{\theta}}^{*(k)}\right]=-N \log (2 \pi)-\frac{N}{2} \log (\psi) \\
-\frac{1}{2 \psi}\left\{\sum_{i=1}^{N_{1}}\left(y_{i 1}-\mathbf{x}_{i}^{\top} \boldsymbol{\beta}\right)^{2}+\sum_{i=N_{1}+1}^{N}\left(\hat{v}_{1 m}^{(k)}-2 \hat{\alpha}_{1 m}^{(k)} \mathbf{x}_{i}^{\top} \boldsymbol{\beta}+\boldsymbol{\beta}^{\top} \mathbf{x}_{i} \mathbf{x}_{i}^{\top} \boldsymbol{\beta}\right)\right\} \\
-\frac{1}{2}\left(1+\frac{\left(\rho^{*}\right)^{2}}{\psi}\right)\left\{\sum_{i=1}^{N_{1}}\left(\hat{v}_{2 o}^{(k)}-2 \hat{\alpha}_{20}^{(k)} \mathbf{w}_{i}^{\top} \boldsymbol{\gamma}+\boldsymbol{\gamma}^{\top} \mathbf{w}_{i} \mathbf{w}_{i}^{\top} \gamma\right)\right. \\
\left.+\sum_{i=N_{1}+1}^{N}\left(\hat{v}_{2 m}^{(k)}-2 \hat{\alpha}_{2 m}^{(k)} \mathbf{w}_{i}^{\top} \boldsymbol{\gamma}+\boldsymbol{\gamma}^{\top} \mathbf{w}_{i} \mathbf{w}_{i}^{\top} \gamma\right)\right\}
\end{gathered}
$$




$$
\begin{aligned}
+\frac{\rho^{*}}{\psi}\left\{\sum_{i=1}^{N_{1}}\left(y_{i 1} \hat{\alpha}_{2 o}^{(k)}-y_{i 1} \mathbf{w}_{i}^{\top} \gamma-\hat{\alpha}_{2 o}^{(k)} \mathbf{x}_{i}^{\top} \boldsymbol{\beta}+\boldsymbol{\beta}^{\top} \mathbf{x}_{i} \mathbf{w}_{i}^{\top} \gamma\right)\right. \\
\left.\sum_{i=N_{1}+1}^{N}\left(\hat{\alpha}_{12 m}^{(k)}-\hat{\alpha}_{1 m}^{(k)} \mathbf{w}_{i}^{\top} \gamma-\hat{\alpha}_{2 m}^{(k)} \mathbf{x}_{i}^{\top} \boldsymbol{\beta}+\boldsymbol{\beta}^{\top} \mathbf{x}_{i} \mathbf{w}_{i}^{\top} \gamma\right)\right\},
\end{aligned}
$$

where specific vectors are provided in the Appendix.

The ECM(NR) algorithm is developed based on the former ECM algorithm to accelerate the convergence process. The only difference is $\hat{\psi}^{*(k+1)}$ in the CM-steps (Conditional Maximization-steps ) is first updated using the Newton-Raphson method, and $\hat{\psi}^{(k+1)}$ is then obtained. The specific expressions can be obtained in Zhao et al. (2020). For a better understanding of the ECM algorithm, Meng and Rubin (1993) and McLachlan and Krishnan (2008) can be referred to.

\section{ECME algorithm}

In this section, the ECME algorithm is briefly introduced and further developed to save running time. Liu and Rubin (1994) and McLachlan and Krishnan (2008) can be referred to in understanding the ECME algorithm in detail.

Assume that the complete data is $\left(\mathbf{z}, \mathbf{y}_{o b s}, \mathbf{u}\right)$, where $\mathbf{z}=\left(z_{1}, \cdots, z_{N_{1}}\right)^{\top}$ is missing data, $\mathbf{y}_{o b s}=$ $\left(y_{11}, \cdots, y_{N_{1}, 1}\right)^{\top}$, and $\mathbf{u}=\left(u_{1}, \cdots, u_{N}\right)^{\top}$. The related parameters $\boldsymbol{\theta}=\{\boldsymbol{\beta}, \boldsymbol{\gamma}, \sigma, \rho\}$ are transformed to $\boldsymbol{\theta}^{*}=\left\{\boldsymbol{\beta}, \gamma, \psi^{*}, \rho^{*}\right\}$ like those in ECM algorithm. The complete data log-likelihood function can be written as

$$
\begin{gathered}
l_{c}\left(\boldsymbol{\theta}^{*} ; \mathbf{z}, \mathbf{y}_{o b s}, \mathbf{u}\right)=-\frac{1}{2} \sum_{i=1}^{N} u_{i} \log (2 \pi \psi)-\sum_{i=1}^{N} \frac{u_{i}\left(y_{i 1}-\mathbf{x}_{i}^{\top} \boldsymbol{\beta}-\rho \sigma z_{i}\right)^{2}}{2 \psi} \\
-\frac{1}{2} \sum_{i=1}^{N} u_{i} \log (2 \pi)-\frac{1}{2} \sum_{i=1}^{N} u_{i} z_{i}^{2}+\sum_{i=1}^{N}\left(1-u_{i}\right) \log \left(\Phi\left(-\mathbf{w}_{i}^{\top} \gamma\right)\right),
\end{gathered}
$$

and the following is the Q-function (which is the conditional expectation of the complete-data loglikelihood $l_{c}\left(\boldsymbol{\theta}^{*} ; \mathbf{z}, \mathbf{y}_{\text {obs }}, \mathbf{u}\right)$ with respect to the conditional distribution of $\mathbf{z}$ given $\mathbf{y}_{\text {obs }}$ and $\left.\mathbf{u}\right)$ calculated at the $k$-th iteration of the E-step:

$$
\begin{gathered}
Q\left(\boldsymbol{\theta}^{*} \mid \hat{\boldsymbol{\theta}}^{*(k)}\right)=E\left[l_{\mathcal{c}}\left(\boldsymbol{\theta}^{*} \mid \mathbf{z}, \mathbf{y}_{o b s}, \mathbf{u}\right) \mid \hat{\boldsymbol{\theta}}^{*(k)}\right]=-\frac{1}{2} \sum_{i=1}^{N} u_{i} \log (2 \pi \psi) \\
-\frac{1}{2 \psi} \sum_{i=1}^{N}\left\{u_{i}\left(y_{i 1}-\mathbf{x}_{i}^{\top} \boldsymbol{\beta}\right)^{2}-2 u_{i}\left(y_{i 1}-\mathbf{x}_{i}^{\top} \boldsymbol{\beta}\right) \rho^{*} \hat{\alpha}_{i}^{(k)}+u_{i}\left(\rho^{*}\right)^{2} \hat{\delta}_{i}^{(k)}\right\} \\
-\frac{1}{2} \sum_{i=1}^{N} u_{i} \log (2 \pi)-\frac{1}{2} \sum_{i=1}^{N} u_{i} \hat{\delta}_{i}^{(k)}+\sum_{i=1}^{N}\left(1-u_{i}\right) \log \left(\Phi\left(-\mathbf{w}_{i}^{\top} \gamma\right)\right),
\end{gathered}
$$

where

$$
\begin{aligned}
& \hat{\alpha}_{i}^{(k)}=E\left[Z_{i} \mid \hat{\theta}^{*^{(k)}}, y_{i 1}, U_{i}=1\right] \text { and } \\
& \hat{\delta}_{i}^{(k)}=E\left[Z_{i}^{2} \mid \hat{\theta}^{*(k)}, y_{i 1}, U_{i}=1\right],
\end{aligned}
$$

with the conditional distribution

$$
Z_{i} \mid \hat{\theta}^{*(k)}, y_{i 1}, U_{i}=1 \sim T N_{\left(-\mathbf{w}_{i}^{\top} \hat{\gamma}^{(k)}, \infty\right)}\left(\frac{\hat{\rho}^{(k)}}{\hat{\sigma}^{(k)}}\left(y_{i 1}-\mathbf{x}_{i}^{\top} \hat{\boldsymbol{\beta}}^{(k)}\right), 1-\hat{\rho}^{2^{(k)}}\right),
$$

where the stochastic representation of the density function for $y_{i 1} \mid u_{i}=1$ is considered. The ECME algorithm is time-consuming because calculating $\hat{\gamma}^{(k+1)}$ requires a significant amount of time. The Newton-Raphson method is applied to reduce the computing time. In the CM-step, the $\hat{\gamma}^{(k)}$ is updated by

$$
\hat{\boldsymbol{\gamma}}^{(k+1)}=\hat{\boldsymbol{\gamma}}^{(k)}-\left[\frac{\partial^{2}}{\partial \gamma \partial \gamma^{\top}} \log L\left(\boldsymbol{\theta}^{*} \mid \hat{\boldsymbol{\theta}}^{*(k)}\right)\right]^{-1} \frac{\partial}{\partial \gamma} \log L\left(\boldsymbol{\theta}^{*} \mid \hat{\boldsymbol{\theta}}^{*(k)}\right),
$$


where

$$
\frac{\partial}{\partial \gamma} \log L\left(\boldsymbol{\theta}^{*} \mid \hat{\boldsymbol{\theta}}^{*(k)}\right)=\sum_{i=1}^{N} u_{i} \frac{\phi(A)}{\Phi(A)} \frac{\hat{\sigma}^{(k+1)}}{\sqrt{\exp \left(\hat{\psi}^{*(k+1)}\right)}} \mathbf{w}_{i}+\sum_{i=1}^{N}\left(u_{i}-1\right) \frac{\phi\left(-\mathbf{w}_{i}^{\top} \gamma^{(k+1)}\right)}{\Phi\left(-\mathbf{w}_{i}^{\top} \gamma^{(k+1)}\right)} \mathbf{w}_{i},
$$

and

$$
\begin{gathered}
\frac{\partial^{2} \log L\left(\boldsymbol{\theta}^{*} \mid \hat{\boldsymbol{\theta}}^{*(k)}\right)}{\partial \gamma \partial \gamma^{\top}}=-\sum_{i=1}^{N} \frac{u_{i} \hat{\sigma}^{(k+1)^{2}}}{\exp \left(\hat{\psi}^{*(k+1)}\right)}\left[A \frac{\phi(A)}{\Phi(A)}+\left(\frac{\phi(A)}{\Phi(A)}\right)^{2}\right] \mathbf{w}_{i} \mathbf{w}_{i}^{\top} \\
+\sum_{i=1}^{N}\left(1-u_{i}\right)\left[\frac{\mathbf{w}_{i}^{\top} \gamma \phi\left(-\mathbf{w}_{i}^{\top} \gamma^{(k+1)}\right)}{\Phi\left(-\mathbf{w}_{i}^{\top} \gamma^{(k+1)}\right)}-\left(\frac{\phi\left(-\mathbf{w}_{i}^{\top} \gamma^{(k+1)}\right)}{\Phi\left(-\mathbf{w}_{i}^{\top} \gamma^{(k+1)}\right)}\right)^{2}\right] \mathbf{w}_{i} \mathbf{w}_{i}^{\top},
\end{gathered}
$$

with

$$
A=\frac{\hat{\sigma}^{(k+1)} \mathbf{w}_{i}^{\top} \hat{\gamma}^{(k)}+\hat{\rho}^{(k+1)}\left(y_{i 1}-\mathbf{x}_{i}^{\top} \hat{\boldsymbol{\beta}}^{(k+1)}\right)}{\sqrt{\exp \left(\hat{\psi}^{*(k+1)}\right)}} .
$$

\section{Implementation in EMSS}

The package EMSS is constructed to describe the three EM-type algorithms. In EMSS, the main function for the estimation of the Heckman selection model is EMSS. A formula for the response equation whose argument is response and a formula for the selection equation with argument selection are required. With the default estimation method ECM ("ECM"), the user can also choose the method "ECMnr" for the ECM(NR) method or "EMCE" for the ECME method. The argument initial. param can be used to set the initial values. If the initial values are not provided by the user, EMSS conducts the estimation of the consistent initial values offered by the two-step method through the package sampleSelection.

The result of EMSS is a list of class 'EMSS', and several methods for the objects of this class are also provided by the package EMSS. Command print prints the estimation results. Command summary calculates and prints the summarized results. coef extracts the estimated coefficients, and vcov extracts the variance-covariance matrix. confint can be used to calculate the confidence intervals of all the parameters by applying the following equation.

$$
\left[\text { pâra }+Z_{\alpha} \times \text { stdrr pâra }+Z_{1-\alpha} \times \text { stdrr }\right],
$$

where pâra is the estimated value of a parameter, stdrr is the corresponding standard error value, and $Z_{\alpha}$ and $Z_{1-\alpha}$ are the quantile values of standard normal distribution at $\alpha$ and $1-\alpha$, respectively, with $\alpha=(1-$ level $) / 2$, where "level" is the confidence level. The default confidence level (level) is 0.95 $(95 \%)$, and it can be changed to any value between 0 and 1 .

\section{Using EMSS}

This section illustrates the usage of EMSS using a simulation example and application to a real data set. An example using random numbers is given first, with exclusion restriction where the two observed characters $\mathbf{X}$ and $\mathbf{W}$ are not the same.

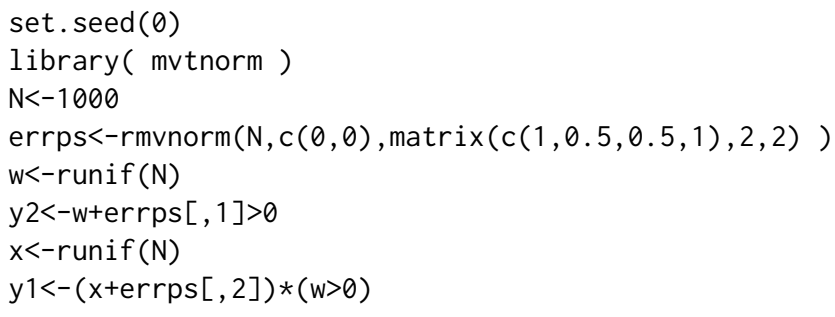

The package mvtnorm is used to create bivariate normal disturbances with a correlation of 0.5 . The observed character for selection, w, is generated by uniform distribution, and the selection outcome $\mathrm{y} 2$ is then generated using the probit generating process. Through a similar process, the explanatory 
variable $x$ and the outcome variable of interest $y 1$ are generated. Note that the two observed characters, $w$ and $x$, are independent and thus fulfill the exclusion restriction. Hence, the parameters $\beta$ and $\gamma$ are set equally as $(0,1)^{\top}$ and must be estimated. The estimated results in the ECM algorithm are as follows.

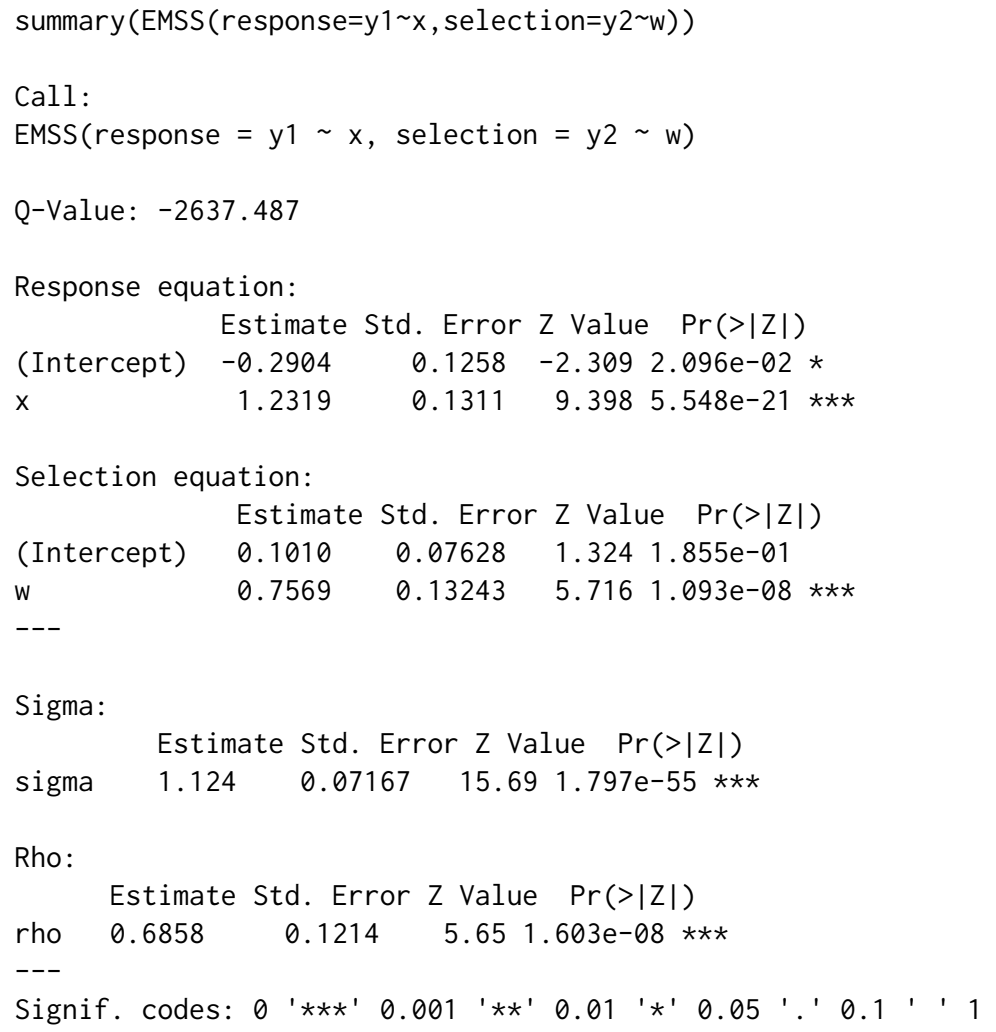

Rho:

The estimated results for the parameters are reasonably precise.

The following real data example is an example in Cameron and Trivedi (2009, Section 16.6.5, p. 546) regarding ambulatory expenditures from the 2001 Medical Expenditure Panel Survey. The data consist of 3328 observations with 526 corresponding to zero expenditures and is available in MEPS2001 of the R package ssmrob. To estimate an individual's medical expenditures, the outcome (response) variable of interest, log ambulatory expenditures (lnambx), is modeled by individual's age (age), gender (female), education attainment in years (educ), ethnicity (blhisp), number of chronic diseases (totchr), and insurance status (ins). The selection variable, ambulatory expenditures, which is described by dambexp is modeled by all the former regressors and the income variable (income). The model is estimated using the ECM(NR) method.

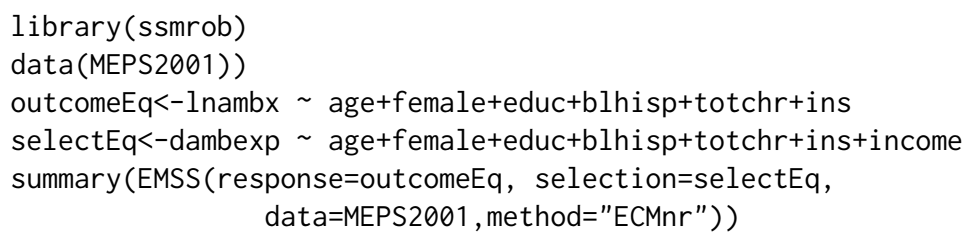

Call:

EMSS (response $=$ outcomeEq, selection $=$ selectEq, data $=$ MEPS2001, method = "ECMnr")

Q-Value: -10213.94

Response equation:

Estimate Std. Error Z Value $\operatorname{Pr}(>|Z|)$

(Intercept) $5.04406 \quad 0.22813 \quad 22.1112 .493 \mathrm{e}-108 * * *$

$\begin{array}{lllll}\text { age } & 0.21197 & 0.02301 & 9.213 & 3.160 \mathrm{e}-20 * * *\end{array}$

$\begin{array}{lllll}\text { femaleTRUE } & 0.34814 & 0.06011 & 5.791 & 6.984 \mathrm{e}-09 * * *\end{array}$

$\begin{array}{lllll}\text { educ } & 0.01872 & 0.01055 & 1.774 & 7.599 \mathrm{e}-02\end{array}$.

blhispTRUE $\quad-0.21857 \quad 0.05967 \quad-3.663 \quad 2.492 \mathrm{e}-04 * * *$ 


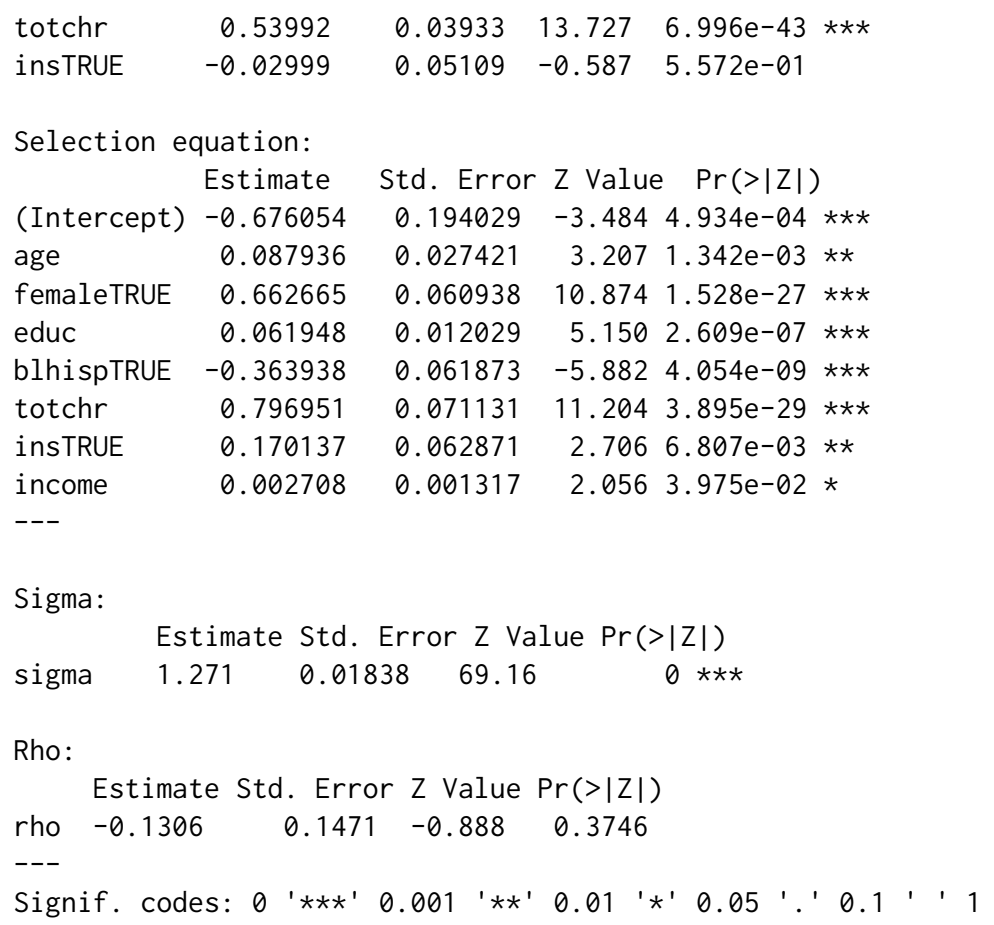

All coefficients and standard errors are completely identical to the results reported in Cameron and Trivedi (2009, Section 16.6.5, p. 546).

The confidence interval of this example can be calculated using the following codes.

$$
\begin{gathered}
\operatorname{confint}(\text { EMSS (response=outcomeEq, selection=selectEq, } \\
\text { data=MEPS2001, method="ECMnr")) }
\end{gathered}
$$

To compare the computing times of the original ECME algorithm and the developed one for which the Newton-Raphson method is applied, the real data example in Section 6, Zhao et al. (2020), is analyzed again. The developed ECME algorithm is used as follows.

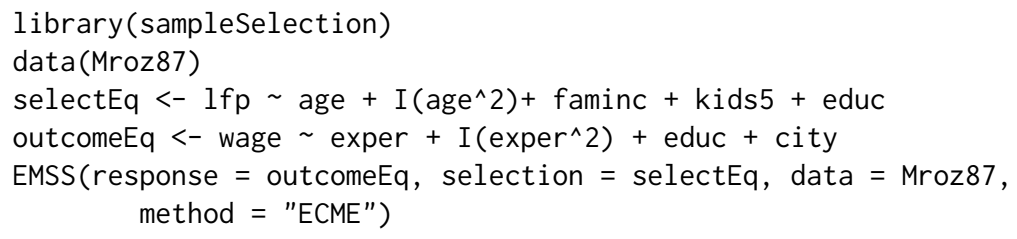

The results are similar to those of the ECM and ECM(NR) algorithm in table 8 of Zhao et al. (2020), which are slightly better than those in the original ECME algorithm.

The EMSS package and R codes were executed on a computer with an Intel(R) Core (TM) i7-4790M CPU at $3.60 \mathrm{GHz}$, running MS-Windows 10. The ECME algorithm developed herein takes $22.67 \mathrm{~s}$ while the original one takes $14.63 \mathrm{~min}$. The computing time of the ECME algorithm is thus significantly reduced than before.

\section{Robustness issues}

Zhao et al. (2020) concluded that a robustness issue arises from the initial values in the MLE method but not in the three EM-type algorithms. Here, we aim to discuss this robustness issue further. For the simulated example in Section Using EMSS, the MLE method is also applied to estimate the data set using the command selection() in R package sampleSelection (Henningsen et al., 2019). The initial values are found to influence the estimated values of parameters in the MLE method. For example, if the initial value of $\sigma$ is set to 5 , that of $\rho$ is set to 0.8 , and those of other parameters are set to 0 s, the results of the MLE method are given as follows.

summary (selection $(y 2 \sim w, y 1 \sim x, \operatorname{star} t=c(\operatorname{rep}(0,4), 5,0.8))$, method="ml")

Tobit 2 model (sample selection model)

Maximum Likelihood estimation 
Newton-Raphson maximization, 3 iterations

Return code 3: Last step could not find a value above the current.

Boundary of parameter space?

Consider switching to a more robust optimization method temporarily.

Log-Likelihood: -2214.037

1000 observations (318 censored and 682 observed)

6 free parameters $(\mathrm{df}=994)$

Probit selection equation:

Estimate Std. Error $t$ value $\operatorname{Pr}(>|t|)$

$\begin{array}{lllll}\text { (Intercept) } & 0.05991 & 0.03482 & 1.721 & 0.0856\end{array}$

$\begin{array}{lllll}\text { w } & 0.03362 & 0.05204 & 0.646 & 0.5185\end{array}$

Outcome equation:

Estimate Std. Error $t$ value $\operatorname{Pr}(>|t|)$

$\begin{array}{lllll}(\text { Intercept })-0.02336 & 0.05811 & -0.402 & 0.688\end{array}$

$\begin{array}{lllll}x & -0.01041 & 0.22132 & -0.047 & 0.963\end{array}$

Error terms:

Estimate Std. Error $t$ value $\operatorname{Pr}(>|t|)$

sigma 5.0000 NA NA NA

$\begin{array}{llll}\text { rho } & 0.9652 & \text { NA } & \text { NA }\end{array}$

Warning messages:

1: In sqrt(diag( $(v c))$ : NaNs produced

2: In sqrt(diag( $(v c))$ : NaNs produced

3: In $\operatorname{sqrt}(\operatorname{diag}(\operatorname{vcov}($ object, part $=$ "full"))) : NaNs produced

The $p$-values of the estimated parameters (except $\sigma$ and $\rho$ ) suggest that the estimated results are not significant. The occurrence of "NA" resulting from the variance-covariance matrix further implies that the estimated results in the MLE method are not reasonable. The previous section, Using EMSS, presents the results from the ECM algorithm where the initial values are set by default based on the results from the two-step method. With the different initial value sets in this section, the ECM, ECM(NR), and ECME algorithms perform stably, and their results are the same as the ones of the ECM algorithm shown in the Using EMSS section to eight decimal places. If the simulated annealing maximizer is applied to select the initial values for the MLE methods, the estimated results are similar to those in the three EM-type algorithms.

To avoid the occasion of the robustness issue in the MLE method, the former scenario is regenerated 1000 times with the degree of censoring corresponding to approximately $30 \%$. Considering the same initial value set as the former, the boxplots in Figures 1 and 2 suggest that the three EM-type algorithms perform similarly and even much better than the MLE algorithm.

In Cameron and Trivedi (2005), the data set RanHIE, which is available in package sampleSelection, based on the "RAND Health Insurance Experiment" is used to analyze how the patient's use of health services is affected by the types of randomly assigned health insurance. An example based on the analysis in Cameron and Trivedi (2005, p. 553) is provided to further discuss this situation.

The outcome variable $\mathbf{y}_{1}$ is lnmeddol, which measures the log of an individual's medical expenses, and the selection variable $\mathbf{y}_{2}$ is binexp, which indicates whether the medical expenses are positive. The observed character $\mathbf{X}$ consists of the $\log$ of the coinsurance rate plus $1(\log c=\log (\operatorname{coins}+1))$, the dummy for the individual deductible plan (idp), the log of participation incentive payment (lpi), the number of chronic diseases (disea), the log of family size (l fam), education of household head in years (educdec), age of individual in years (xage), quadratic polynomial in the age of individual in years, and a dummy variable for female individuals (female). The observed character $\mathbf{W}$ consists of logc, physical limitations (phslm), disea, quadratic polynomial in disea, lfam, educdec, xage, and female. A partial sample where the study year (year) is equal to 2 and the education information is given is selected for the estimation with sample size $N=5,574$.

Fix the initial values of all parameters except $\sigma$ and $\rho$ at 0 and consider three different initial values sets for $\sigma$ and $\rho$. If the initial values of $\sigma$ range from 0.1 to 0.65 , then the estimated values of all parameters in the MLE method are the corresponding initial values regardless of the initial values of $\rho$. The estimated results in the ECM algorithm are stable as follows.

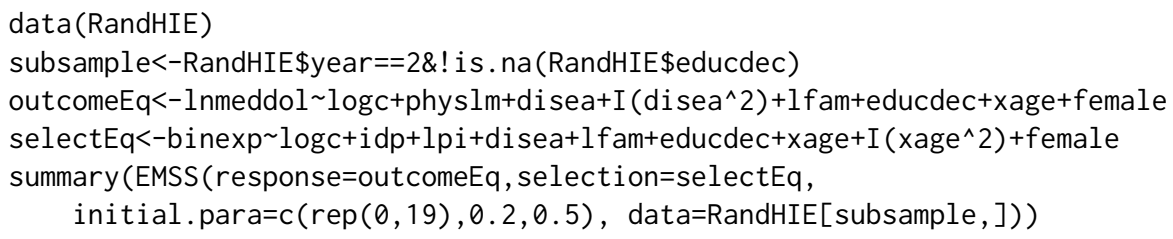


$b_{0}$

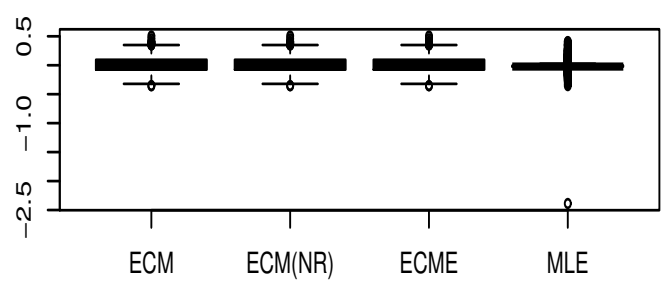

90
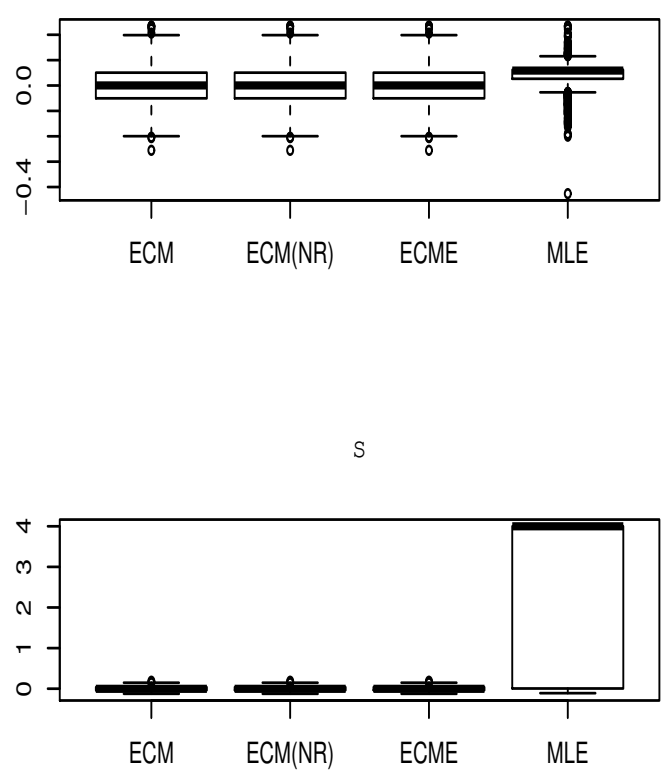

$b_{1}$

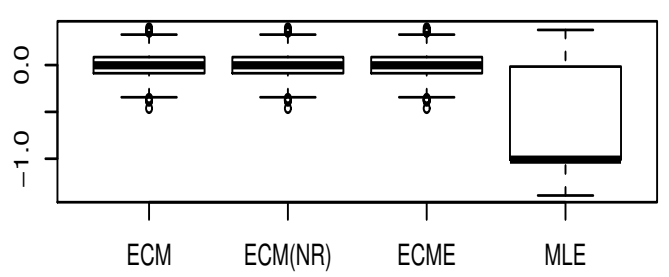

9
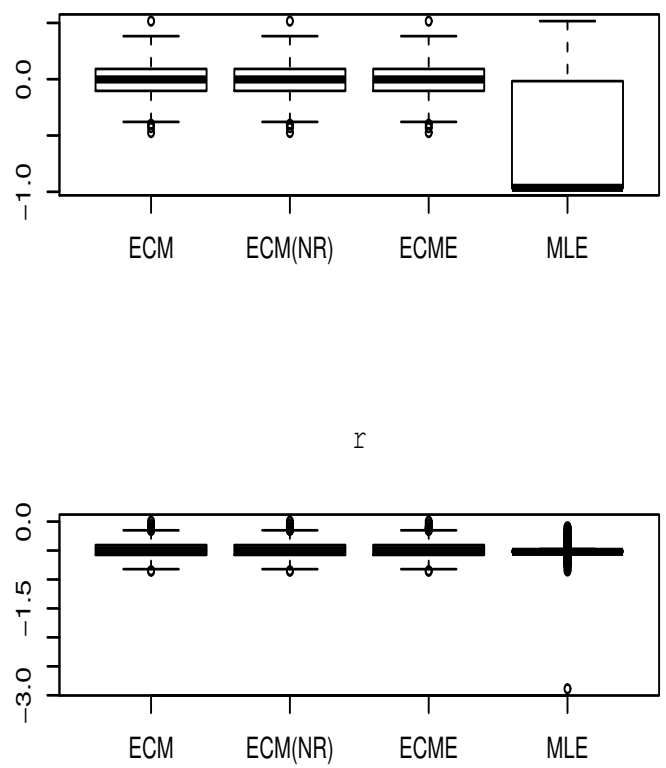

Figure 1: Boxplots of the bias in the estimation for the 1000 regeneration of the simulated example in the section Using EMSS. 
$b_{0}$

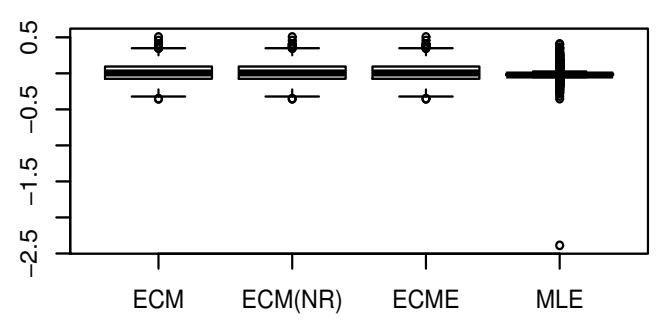

90
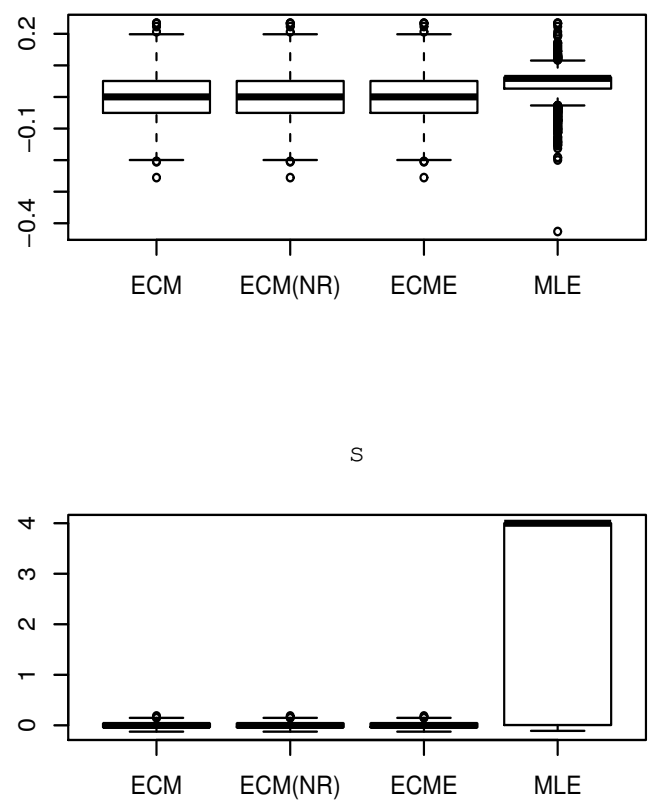

$\mathrm{b}_{1}$

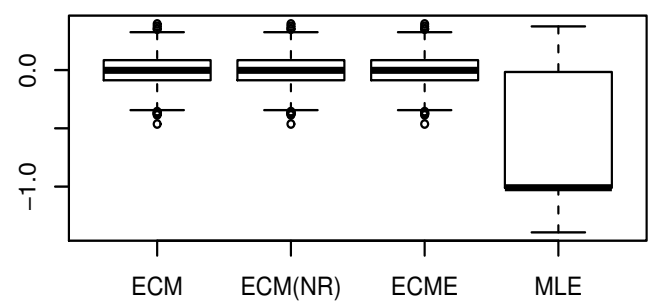

9

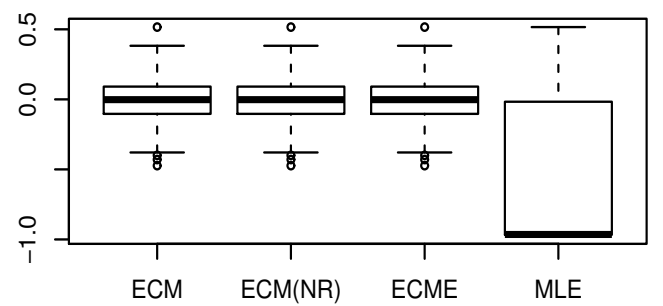

r

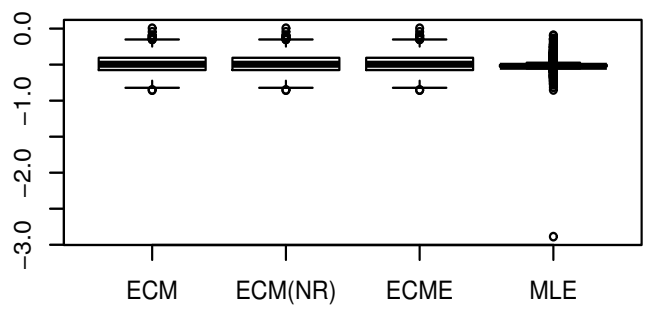

Figure 2: Boxplots of the MSE in the estimation for the 1000 regeneration of the simulated example in the section Using EMSS. 
Call:

EMSS (response $=$ outcomeEq, selection = selectEq,

data $=$ RandHIE[subsample, $]$ )

Q-Value: -16195.75

Response equation:

Estimate Std. Error $Z$ Value $\operatorname{Pr}(>|Z|)$

$\begin{array}{lllll}\text { (Intercept) } & 2.4841461 & 0.168714 & 14.7240 & 4.523 \mathrm{e}-49\end{array} * * *$

logc $\quad-0.1199851 \quad 0.011946-10.0440 \quad 9.762 \mathrm{e}-24 * * *$

physlm $\quad 0.2952680 \quad 0.068552 \quad 4.3072 \quad 1.653 \mathrm{e}-05 * * *$

$\begin{array}{lllll}\text { disea } \quad 0.0415756 & 0.008574 & 4.8490 & 1.241 \mathrm{e}-06 & * * *\end{array}$

$\begin{array}{lllll}\text { I (disea^2) } & -0.0001355 & 0.000250 & -0.5421 & 5.878 \mathrm{e}-01\end{array}$

$\begin{array}{lllll}\text { If am } & -0.1828111 & 0.048101 & -3.8006 & 1.443 \mathrm{e}-04 * * *\end{array}$

$\begin{array}{lllll}\text { educdec } & 0.0350172 & 0.008674 & 4.0368 & 5.418 \mathrm{e}-05\end{array} * * *$

$\begin{array}{lllll}\text { xage } \quad 0.0203750 & 0.001588 & 12.8310 & 1.100 \mathrm{e}-37 * * *\end{array}$

female $\quad 0.3123718 \quad 0.048632 \quad 6.4231 \quad 1.335 \mathrm{e}-10 * * *$

Selection equation:

Estimate Std. Error $\mathrm{Z}$ Value $\operatorname{Pr}(>|\mathrm{Z}|)$

(Intercept) $-0.0807292 \quad 1.240 \mathrm{e}-01 \quad-0.651 \quad 5.150 \mathrm{e}-01$

logc $\quad-0.1138537 \quad 1.078 \mathrm{e}-02-10.5624 .470 \mathrm{e}-26$ ***

idp $\quad-0.0632783 \quad 3.994 \mathrm{e}-02 \quad-1.584 \quad 1.131 \mathrm{e}-01$

lpi $\quad 0.0320468 \quad 7.301 \mathrm{e}-03 \quad 4.3891 .136 \mathrm{e}-05 * * *$

disea $\quad 0.0283038 \quad 3.329 \mathrm{e}-03 \quad 8.503 \quad 1.841 \mathrm{e}-17 * * *$

Ifam $\quad-0.0666747 \quad 3.799 \mathrm{e}-02 \quad-1.755 \quad 7.922 \mathrm{e}-02$.

educdec $\quad \begin{array}{lllll}0.0516196 & 6.923 \mathrm{e}-03 & 7.456 & 8.928 \mathrm{e}-14\end{array}$ ***

$\begin{array}{lllll}\text { xage } \quad-0.0051879 & 4.066 \mathrm{e}-03 & -1.276 & 2.020 \mathrm{e}-01\end{array}$

$\begin{array}{lllll}\text { I (xage^2) } \quad 0.0001979 & 6.907 \mathrm{e}-05 & 2.865 & 4.164 \mathrm{e}-03 * *\end{array}$

female $\quad 0.2098103 \quad 3.829 \mathrm{e}-02 \quad 5.4794 .281 \mathrm{e}-08$ ***

Sigma:

Estimate Std. Error $Z$ Value $\operatorname{Pr}(>|Z|)$

$\begin{array}{lllll}\text { sigma } & 1.604 & 0.02888 & 55.56 & 0 * * *\end{array}$

Rho:

Estimate Std. Error Z Value $\operatorname{Pr}(>|Z|)$

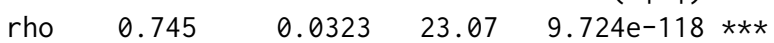

Signif. codes: 0 '***' 0.001 '**' 0.01 '*' 0.05 '.' 0.1 ' ' 1

In the second initial value set, the initial value of $\rho$ is 0.5 , and that of $\sigma$ is within $[0.66,7.9]$. The three new EM-type algorithms perform similarly to the ECM algorithm in the first initial value set. The estimated results in the MLE method are stable like those in the ECM algorithm.

In the third initial value set, the initial values of $\sigma$ are larger than 7.9, and the three new EM-type algorithms still perform stably, similar to the former. However, the estimated values of each parameter in the MLE method are not stable. For example,

summary (selection (selectEq, outcomeEq, $\operatorname{start}=c(\operatorname{rep}(0,19), 8.8,0.5)$, data=RandHIE[subsample, ], method="ml" ))

Tobit 2 model (sample selection model)

Maximum Likelihood estimation

Newton-Raphson maximization, 3 iterations

Return code 3: Last step could not find a value above the current.

Boundary of parameter space?

Consider switching to a more robust optimization method temporarily.

Log-Likelihood: -15707.81

5574 observations (1293 censored and 4281 observed)

21 free parameters $(\mathrm{df}=5553)$

Probit selection equation:

$$
\text { Estimate Std. Error } t \text { value } \operatorname{Pr}(>|t|)
$$

$\begin{array}{lllll}\text { (Intercept) } & 2.155 \mathrm{e}-03 & 1.029 \mathrm{e}-01 & 0.021 & 0.9833\end{array}$ 


\begin{tabular}{|c|c|c|c|c|}
\hline $\log c$ & $5.082 e-03$ & $8.366 \mathrm{e}-03$ & 0.607 & 0.5436 \\
\hline idp & $1.106 \mathrm{e}-03$ & $2.536 \mathrm{e}-02$ & 0.044 & 0.9652 \\
\hline lpi & $6.242 \mathrm{e}-04$ & $4.436 \mathrm{e}-03$ & 0.141 & 0.8881 \\
\hline disea & $-4.776 e-03$ & $2.504 \mathrm{e}-03$ & -1.907 & 0.0565 \\
\hline lfam & $4.923 e-03$ & $3.258 \mathrm{e}-02$ & 0.151 & 0.8799 \\
\hline educdec & $-1.921 e-03$ & $5.710 \mathrm{e}-03$ & -0.336 & 0.7366 \\
\hline xage & $-2.601 e-03$ & $2.440 \mathrm{e}-03$ & -1.066 & 0.2866 \\
\hline$I\left(\right.$ xage $\left.^{\wedge} 2\right)$ & $-1.355 e-05$ & $3.918 \mathrm{e}-05$ & -0.346 & 0.7294 \\
\hline female & $-2.802 e-03$ & $3.258 \mathrm{e}-02$ & -0.086 & 0.9315 \\
\hline \multicolumn{5}{|c|}{ Outcome equation: } \\
\hline & Estimate & Std. Error & $t$ value & $\operatorname{Pr}(>|t|)$ \\
\hline (Intercept) & -0.0056280 & 0.8243005 & -0.007 & 0.99455 \\
\hline $\log c$ & -0.0120012 & 0.0616586 & -0.195 & 0.84568 \\
\hline physlm & 0.0001365 & 0.2694167 & 0.001 & 0.99960 \\
\hline disea & -0.0359361 & 0.0349727 & -1.028 & 0.30421 \\
\hline$I($ disea^ 2$)$ & 0.0001022 & 0.0009454 & 0.108 & 0.91390 \\
\hline lfam & -0.0086017 & 0.2567682 & -0.033 & 0.97328 \\
\hline educdec & -0.0622248 & 0.0449205 & -1.385 & 0.16604 \\
\hline xage & -0.0286630 & 0.0081770 & -3.505 & $0.00046 * * *$ \\
\hline female & -0.0023083 & 0.2580752 & -0.009 & 0.99286 \\
\hline \multicolumn{5}{|l|}{ Error terms: } \\
\hline \multicolumn{2}{|c|}{ Estimate Std. } & \multicolumn{3}{|c|}{ Error $t$ value $\operatorname{Pr}(>|t|)$} \\
\hline \multicolumn{2}{|c|}{8.8015} & \multicolumn{3}{|c|}{ NA NA NA } \\
\hline 0.9 & 0.9186 & NA & \multicolumn{2}{|l|}{ NA } \\
\hline
\end{tabular}

Warning messages:

1: In $\operatorname{sqrt}(\operatorname{diag}(\mathrm{vc}))$ : NaNs produced

2: In sqrt(diag(vc)): NaNs produced

3: In sqrt(diag $(\operatorname{vcov}($ object, part $=" f u l l "))):$ NaNs produced

The warning messages, the $p$-value of each parameter, and the "NA" in the standard errors of parameters $\sigma$, and $\rho$ suggest that the estimated results of the MLE method are not reasonable.

By summarizing the above three initial value sets with setting the initial values of $\sigma$ at $\{0.1,0.3, \ldots, 10\}$, Figure 3 presents the histograms of the estimated coefficients for the variables physlm, female, and the estimated values of the parameter $\sigma$ in all four algorithms. The histograms illustrate that the horizontal axis value in the three EM-type algorithms are the same all the time, which further suggests that three EM-type algorithms do not affect by the initial values, and they perform similarly. However, the histograms of the MLE method imply that the MLE method is not stable.

This suggests that the three EM-type algorithms are more robust than the MLE method. However, the computing times of the above three examples using the ECME algorithm are relatively longer than those of ECM and ECM(NR). It is found that the computing time of the ECME algorithm is affected by the sample size. For instance, under different sample sizes, Table 1 presents the running time of the ECM, ECM(NR), and ECME algorithms for the simulated example shown in the section using EMSS with 10-times regeneration. As the sample size increases, the ECME algorithm costs much more time than the ECM and ECM(NR) algorithms.

\begin{tabular}{llllll}
\hline \hline Sample sizes $n$ & 200 & 300 & 500 & 800 & 1000 \\
\hline ECM & 2.5415 & 3.1542 & 4.4769 & 6.1861 & 7.8049 \\
ECM(NR) & 2.5232 & 3.0921 & 4.4393 & 6.0212 & 7.6943 \\
ECME & 7.7486 & 16.9576 & 26.7005 & 119.0904 & 159.1983 \\
\hline \hline
\end{tabular}

Table 1: Running time (seconds) of the simulated example in the section Using EMSS with 10-times regeneration.

If the sample size of the former RandHIE data example decreases to 1,000 randomly with the same outcome and selection models, MLE presents results similar to the three EM-type algorithms only when the initial values of $\sigma$ ranges from 0.71 to 7.0 (the values may change since the sample size is randomly reduced to 1,000). The three EM-type algorithms still present stable results that are similar to each other. Furthermore, the EMCE algorithm takes a similar computing time with the ECM and $\mathrm{ECM}(\mathrm{NR})$. So it will be better to use ECM or ECM(NR) algorithms for the large-size samples.

To achieve more robust estimation, the simulated annealing maximizer for 10,000 iterations is applied to offer better initial values for the MLE method. Note that the selected value for "parscale" in 
By the ECM algorithm

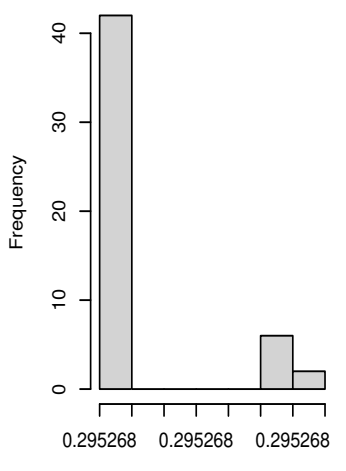

Estimated Coefficients of physlm

By the ECM algorithm

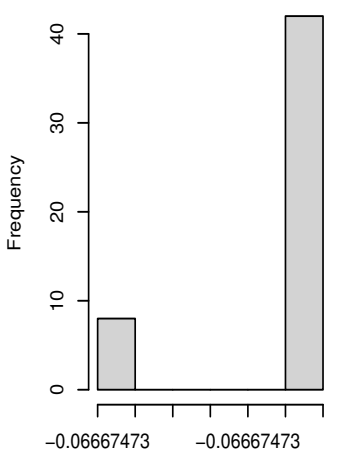

Estimated Coefficients of female

By the ECM algorithm

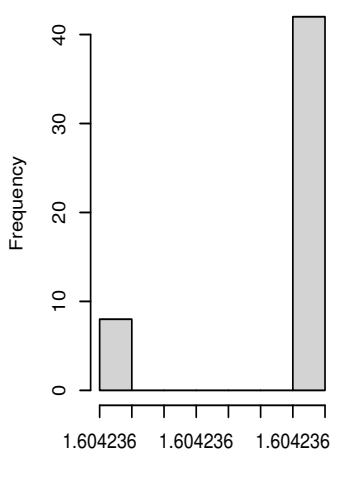

Estimated $\sigma$
By the ECM(NR) algorithm

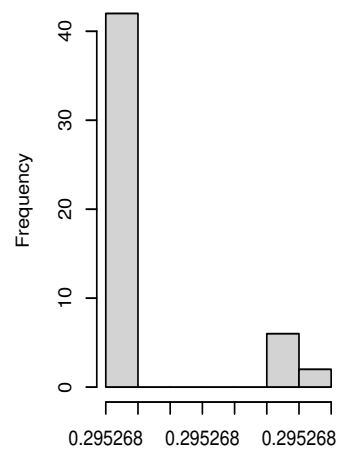

Estimated Coefficients of physlm

By the ECM(NR) algorithm

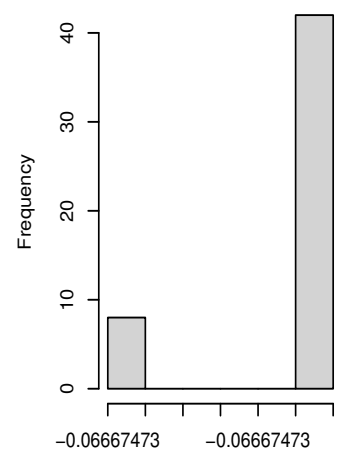

Estimated Coefficients of female

By the ECM(NR) algorithm

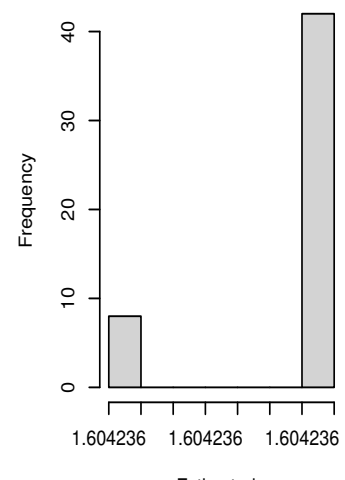

Estimated $\sigma$
By the ECME algorithm

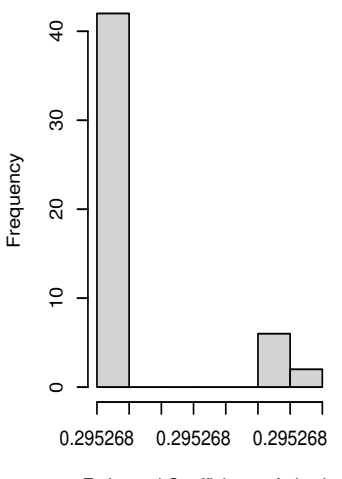

Estimated Coefficients of physlm

By the ECME algorithm

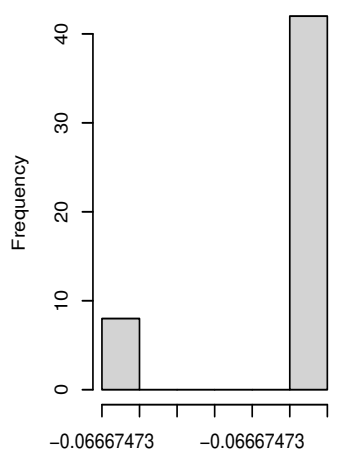

Estimated Coefficients of female

By the ECME algorithm

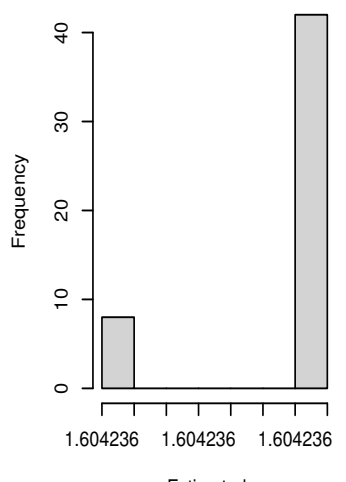

Estimated $\sigma$
By the MLE method

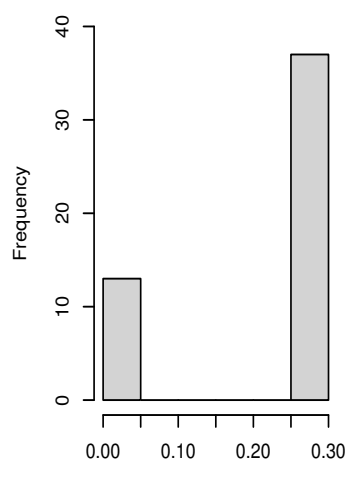

Estimated Coefficients of physlm

By the MLE algorithm

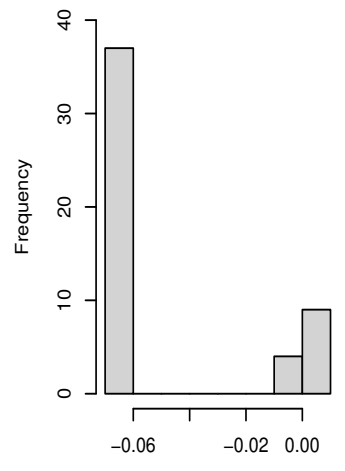

Estimated Coefficients of female

By the MLE algorithm

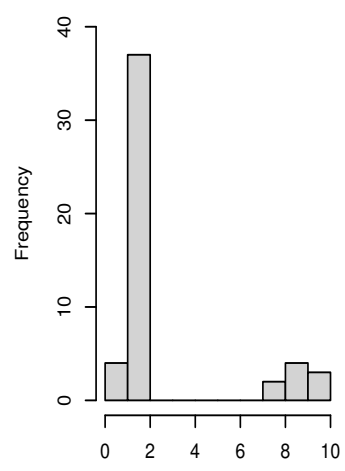

Estimated $\sigma$

Figure 3: Some estimated values in the four algorithms for the RanHIE data. 
SANN is 0.001 , which was satisfactory for this data set. The estimated results of the parameters are the same as those in the MLE method in the second initial value set. The calculated log-likelihood value is -10331.12 , which is greater than -15707.81 in the third initial value set.

\section{Discussion}

The ECME algorithm is developed through the application of the Newton-Raphson method to reduce the computing time. The implementation of three new EM-type algorithms, namely ECM, ECM(NR), and ECME, are described in package EMSS. The application of the package EMSS is conducted using simulated and real data sets. The examples for which initial values are considered in detail further confirm that the three new EM-type algorithms are more robust than the MLE method. The EMSS package is preferable to the sampleSelection when the robustness issue arising from the initial values is involved. The standard errors might not be calculated appropriately in the MLE or the two-step method in the sampleSelection because of the unreasonable results, but they can always be calculated effectively using the EMSS package.

\section{Acknowledgment}

The corresponding author's research was supported by Basic Science Research Program through the National Research Foundation of Korea (NRF) funded by the Ministry of Education (2018R1D1A1B07045603) and the National Research Foundation of Korea (NRF) grant funded by the Korea government (MSIT) (2021R1A4A5032622).

\section{Bibliography}

A. C. Cameron and P. K. Trivedi. Microeconometrics: Methods and Applications. Cambridge University Press, New York, 2005. [p312]

A. C. Cameron and P. K. Trivedi. Microeconometrics using Stata. Stata Press, College Station, Texas, 2009. [p310, 311]

J. Heckman. Shadow prices, market wages, and labor supply. Econometrica, 42(4):679-694, 1974. URL http://www. jstor.org/stable/1913937. [p306, 307]

J. Heckman. Sample selection bias as a specification error. Econometrica, 47(1):153-161, 1979. URL https://www. jstor.org/stable/1912352. [p306]

A. Henningsen, O. Toomet, and S. Petersen. sampleSelection: Sample Selection Models. R Foundation for Statistical Computing, 2019. URL https://cran.r-project.org/web/packages/sampleselection/ index.html. [p311]

R. J. A. Little and D. B. Rubin. Statistical Analysis with Missing Data. Wiley \& Sons, Inc., New Jersey, 2002. doi: 10.1002/9781119013563. [p306]

C. Liu and D. Rubin. The ecme algorithm: A simple extension of em and ecm with faster monotone convergence. Biometrika, 81:633-648, 1994. doi: 10.1093/biomet/81.4.633. [p306, 308]

G. McLachlan and T. Krishnan. The EM Algorithm and Extensions, second ed. Wiley, New Jersey, 2008. [p306, 308]

X. Meng and D. Rubin. Maximum likelihood estimation via the ecm algorithm: A general framework. Biometrika, 80:267—-278, 1993. doi: 10.1093/biomet/80.2.267. [p306, 308]

O. Toomet and A. Henningsen. Sample selection models in r: Package sampleselection. Econometrica, 27(7):1-23, 2008. doi: 10.18637/jss.v027.i07. [p306]

J. Zhao, H.-J. Kim, and H.-M. Kim. New em-type algorithms for the heckman selection model. Computational Statistics and Data Analysis, 146:waiting, 2020. doi: 10.1016/j.csda.2020.106930. [p306, 308, 311]

M. Zhelonkin, M. G. Genton, and E. Ronchetti. Robust inference in sample selection models. Journal of the Royal Statistical Society, Series B, 78:805—827, 2016. doi: 10.1111/rssb.12136. [p306] 
Kexuan Yang

Department of Applied Statistics, Konkuk University

120 Neungdong-ro, Gwangiin-gu, Seoul

South Korea

717260446@qq.com

Sang Kyu Lee

Department of Statistics and Probability, Michigan State University 619 Red Cedar Road East Lansing, MI 48824-1027

the USA

lsk0816@gmail.com

Jun Zhao

School of Mathematics and Statistics, Ningbo University

No. 818, Fenghua Road, Ningbo, Zhejiang

China

zhaojun2021@hotmail.com

Hyoung-Moon Kim

Department of Applied Statistics, Konkuk University

120 Neungdong-ro, Gwangjin-gu, Seoul

South Korea

hmkimakonkuk.ac.kr 


\section{Appendix}

Some vectors in the Q-function of ECM and ECM(NR) algorithms at the $k$-th iteration are presented herewith. For missing $y_{i 1}$,

i) $\hat{\alpha}_{1 m}^{(k)}=E\left(Y_{i 1} \mid Y_{i 2} \leq 0\right)=\hat{\mu}_{i 1}^{(k)}-\hat{\rho}^{(k)} \lambda\left(-\hat{\mu}_{i 2}^{(k)}\right)$, where $\hat{\mu}_{i 1}^{(k)}=\mathbf{x}_{i}^{\top} \hat{\boldsymbol{\beta}}^{(k)}$ and $\hat{\mu}_{i 2}^{(k)}=\mathbf{w}_{i}^{\top} \hat{\gamma}^{(k)}$;

ii) $\hat{\alpha}_{2 m}^{(k)}=E\left(Y_{i 2} \mid Y_{i 2} \leq 0\right)=\hat{\mu}_{i 2}^{(k)}-\lambda\left(-\hat{\mu}_{i 2}^{(k)}\right)$;

iii) $\hat{v}_{1 m}^{(k)}=E\left(Y_{i 1}^{2} \mid Y_{i 2} \leq 0\right)=\hat{\mu}_{i 1}^{2(k)}+\hat{\sigma}^{2(k)}-\hat{\rho}^{*}(k) \lambda\left(-\hat{\mu}_{i 2}^{(k)}\right)\left(2 \hat{\mu}_{i 1}^{(k)}-\hat{\rho}^{*}(k) \hat{\mu}_{i 2}^{(k)}\right)$;

iv) $\hat{v}_{2 m}^{(k)}=E\left(Y_{i 2}^{2} \mid Y_{i 2} \leq 0\right)=1+\hat{\mu}_{i 2}^{2(k)}-\hat{\mu}_{i 2}^{(k)} \lambda\left(-\hat{\mu}_{i 2}^{(k)}\right)$;

v) $\hat{\alpha}_{12 m}^{(k)}=E\left(Y_{i 1} Y_{i 2} \mid Y_{i 2} \leq 0\right)=\hat{\mu}_{i 1}^{(k)}\left(\hat{\mu}_{i 2}^{(k)}-\lambda\left(-\hat{\mu}_{i 2}^{(k)}\right)\right)+{\hat{\rho^{*}}}^{(k)}$,

and for observed $y_{i 1}$,

vi) $\hat{\alpha}_{20}^{(k)}=E\left(Y_{i 2} \mid Y_{i 1}, Y_{i 2}>0\right)=\hat{\mu}_{i 2.1}^{(k)}+\sqrt{1-\hat{\rho}^{2(k)}} \lambda\left(\frac{\hat{\mu}_{i 2.1}^{(k)}}{\sqrt{1-\hat{\rho}^{2(k)}}}\right)$; $\hat{v}_{20}^{(k)}=E\left(Y_{i 2}^{2} \mid Y_{i 1}, Y_{i 2}>0\right)=1-\hat{\rho}^{2(k)}+\hat{\mu}_{i 2.1}^{2(k)}+\hat{\mu}_{i 2.1}^{(k)} \sqrt{1-\hat{\rho}^{2(k)}} \lambda\left(\frac{\hat{\mu}_{i 2.1}^{(k)}}{\sqrt{1-\hat{\rho}^{2(k)}}}\right)$, where $\hat{\mu}_{i 2.1}^{(k)}=$ $\mathbf{w}_{i}^{\top} \gamma^{(k)}+\frac{\hat{\rho}^{(k)}}{\hat{\sigma}^{(k)}}\left(y_{i 1}-\mathbf{x}_{i}^{\top} \boldsymbol{\beta}^{(k)}\right)$. 